\title{
X-Pinches as Broadband Sources of X-Rays for Radiography
}

\author{
Tatiana Shelkovenko, Sergey Pikuz, David Hammer \\ Laboratory of Plasma Studies, Cornell University, Ithaca, NY, USA \\ Email: tc50@cornell.edu,sap17@cornell.edu,dah5@cornell.edu
}

Received 25 September 2015; accepted 6 November 2015; published 9 November 2015

Copyright (C) 2015 by authors and Scientific Research Publishing Inc.

This work is licensed under the Creative Commons Attribution International License (CC BY). http://creativecommons.org/licenses/by/4.0/

c) (i) Open Access

\begin{abstract}
Two methods of using the X-pinch as a source of X-ray radiation for radiography of biological objects are presented. X-pinches are found to be a very flexible method for generation of radiation over a wide spectral range and provide a high spatial and temporal resolution.
\end{abstract}

\section{Keywords}

\section{X-Pinch, X-Ray, Hot Spot, Electron Beam, Point-Projection Radiography, Biological Object}

\section{Introduction}

The X-pinch, first proposed in 1982 at the Lebedev Physical Institute, Russian Academy of Sciences, as a source of hot dense plasma [1], was being actively studied as an interesting object of high energy density physics. An X pinch plasma is generated using two (or more) fine wires being arranged so that they cross and touch at a single point, forming an " $\mathrm{X}$ " shape, as the load of a high current pulsed power generator [1]-[7]. Such generators produce current pulses ranging from $50 \mathrm{kA}$ to $1 \mathrm{MA}$ peak current, or even more, with pulse durations typically in the range 100 to 300 ns. Small, bright, X-ray emitting plasma "hot spots" are reliably formed which is close to the cross point for a wide range of wire materials, wire diameters and current pulse parameters. The wide spectral range of the radiated energy from a tiny volume and a predictable location offer the possibility of using the $\mathrm{X}$-pinch hot spots as sources of X-ray radiation for different applications. In specific cases, the X-pinch provides a very small size $(\sim 1 \mu \mathrm{m})$, short duration ( $<100 \mathrm{ps})$, bright X-ray burst of thermal radiation $(\sim 1 \mathrm{keV})$ for use as an X-ray backlighter for point-projection radiography. Immediately after the thermal X-ray burst is emitted, a gap that increases in just a few ns from tens of $\mu \mathrm{m}$ to $\sim$ several $\mathrm{mm}$ is developed. Energetic electrons are accelerated across that gap, as evidenced by the generation of non-thermal X-ray radiation in the 10 - $150 \mathrm{keV}$ energy range from a 0.1 to $1 \mathrm{~mm}$ source [8]-[10].

The hybrid X-pinch (HXP) configuration consists of a high current diode with conical tungsten electrodes se- 
parated by a $1-3 \mathrm{~mm}$ gap that is shorted by a 20 to $100 \mu \mathrm{m}$ diameter wire. First proposed in 2008, HXPs were now used as point sources of soft X-ray emission in projection radiography and other applications [11]-[13].

The X-pinches in different configurations have proven to be a very flexible source of radiation over a wide spectral range and capable of operating over a wide range of initial conditions. For radiography, the field of view is limited only by the size of the radiation detector (film or an image plate). Unique properties of X-pinches allow one to use them as the probing source in high-resolution projection X-ray imaging of various physical and biological objects.

Results of point-projection radiography of biological objects obtained in the standard X-pinch in the last fifteen years together with new results obtained in the HXPs are presented in the paper.

\section{Method}

A method of point-projection radiography for biological object imaging using X-pinches as a source of a probing radiation in wide energy band is presented and possibilities of the method are discussed.

A schematic diagram of point-projection radiography is shown in Figure 1. This very simple method takes advantage of the small source and short X-ray emission duration of the X-pinch. In this case, it is possible to obtain an object image with a high magnification and a high spatial resolution [3]-[6]. For point-projection X-ray
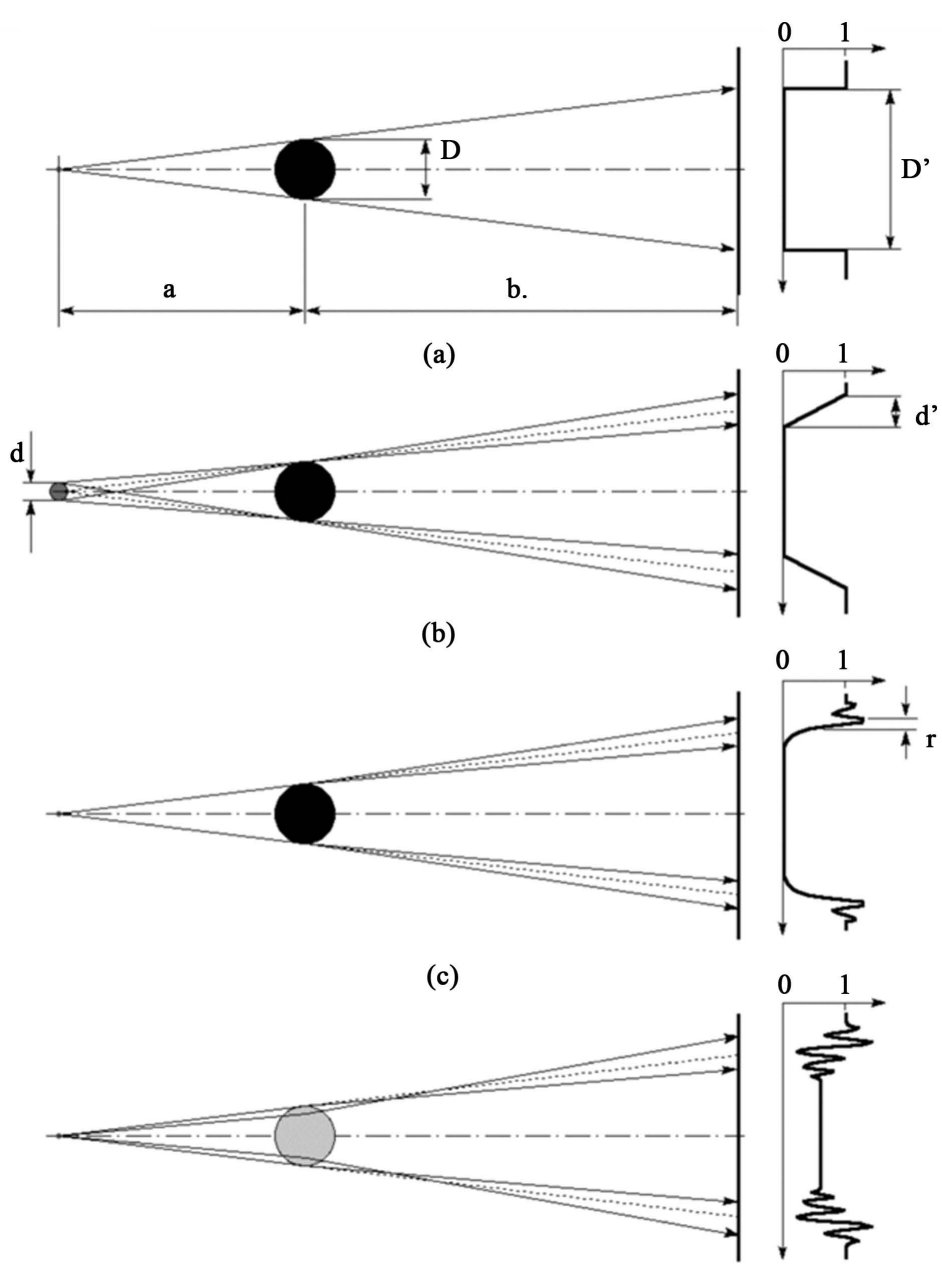

(d)

Figure 1. Illustration of point-projection X-ray radiography (a) using a point source and (b) using a source with diameter d (in the ray optics approximation); illustration of pointprojection X-ray radiography using a point source and taking into account diffraction (c) by an opaque object and (d) by a semitransparent object. 
radiography, the small size of the source is the most important characteristic because it largely determines the spatial resolution of the method (Figure 1(b)). Because of the short wavelength of X-ray radiation, in the majority of cases, the influence of the size of the radiation source on the spatial resolution can be calculated using formulas of geometrical optics, without taking into account the wave factors of the radiation. However, as was shown in refs [14]-[16], the wave properties of the radiation used, i.e. diffraction, refraction and interference of the radiation incident on the object under study, do have a negative effect on the spatial resolution (Figure 1(c) and Figure 1(d)).

The processes listed above will distort the pattern in one way or another, being nevertheless related to the interaction of the radiation from the source with the object and, consequently, carrying information on the object. However, the processing of this information may prove to be a rather complicated task. Therefore, in the first approximation, the processes of diffraction, refraction and interference can be considered factors that degrade the spatial resolution. Upon a decrease in the size of the source to a certain limit, these factors can become very important.

The extremely small size of the source and an increased object-detector distance can lead to a coherence-enhanced (phase contrast) radiograph (as illustrated in Figure 1(c)). A phase contrast image allows interference fringes to develop from the phase distortions during X-ray propagation through the object, providing substantial edge enhancement. Figure 2 shows a comparison of a simple absorption image and a phase contrast image of a spider to show that the phase contrast imaging can help to see the details of the object that are not visible (too small and transparent for the radiation used for radiography) in the case of simple absorption imaging.

Using two or more X-pinches in a high current diode, as illustrated in Figure 3(c), it is possible to get two or more frames in a single test. To simplify X-pinch loading and make X-pinches more predictable the hybrid X-pinch described above and shown in Figure 3(d) was developed and used for radiography [11]-[13].

To record the images, X-ray films, fluorescent imaging plates or CCD-cameras can be used. Most of images presented in the paper were recorded on film because most films have smaller sensitive grain size than the pixels of all other detectors.

In previous X-pinch studies and applications, the high-current generators used to power them are relatively large, albeit simple electrically, which hampers their use in biology and medicine [2] [17]. However, a new generation of pulse generators developed in recent years are simple in design and are much smaller than their predecessors [18]-[20]. These generators are portable and do not require specialized personnel for their operation. Such generators are promising for enabling the use of X-pinch X-ray sources in biology and medicine.

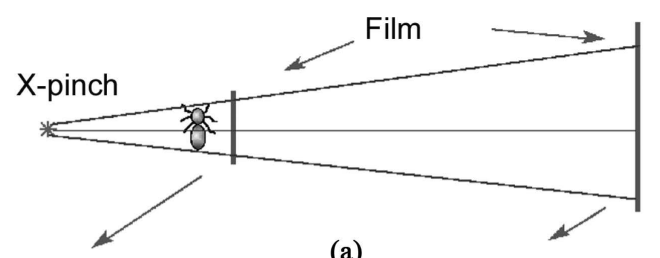

$\mathrm{x} 1.1$

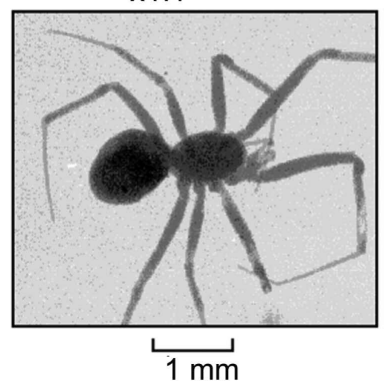

(a)

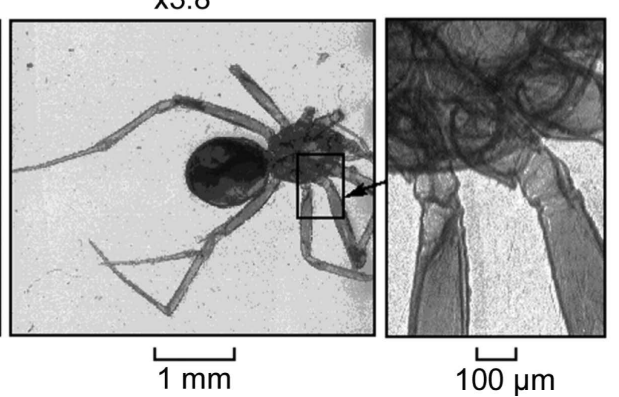

(c)

(d)

Figure 2. (a) Diagram of the point-projection imaging method used in these experiments; (b) simple absorption image; (c) a phase contrast image of a spider (in vacuum) and (d) enlarged part of the image in (c), demonstrating strong phase contrast on the transparent spider legs. The images were obtained in the radiation from a 4-wire Mo X-pinch on the XP generator. 


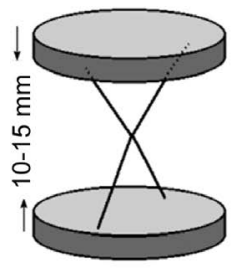

(a)

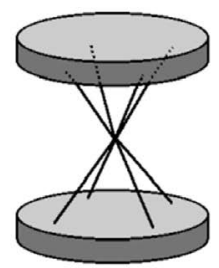

(b)

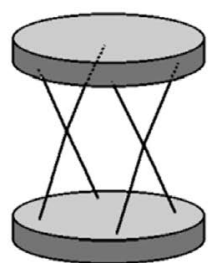

(c)

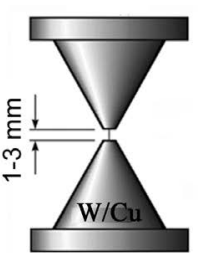

(d)

Figure 3. Two (a) and multi-wire (b) X pinches placed between the output electrodes of a high current pulsed power machine are shown alone. Two X-pinches are shown in parallel in (c) and the hybrid X-pinch configuration is shown in (d).

A standard X-pinch consists of from 2 to 8 wires has been studied as a source of soft X-ray emission in the photon energy range $1-10 \mathrm{keV}$ in considerable detail [1] [3]-[5] [7], including as applied to point-projection X-ray imaging [2] [6] [15] [16]. The radiating X-pinch hot spot region is a high-temperature, near solid density plasma that develops in the micropinch formed at the original wire cross point, as shown in the point-projection $\mathrm{X}$-ray images shown in Figure 4. It is one of the brightest soft X-ray sources emitting in the photon energy range of $1-10 \mathrm{keV}$, and it possesses unique parameters: a micron $(\mu \mathrm{m})$-size radiating region and a picosecond time scale pulse duration [4]-[7] [8]. Such a radiation source is ideal for the method of point-projection X-ray imaging, which is an efficient diagnostic means to obtain both qualitative and quantitative data on the object under study [21].

The hot spot formation is followed by the breaking up of the plasma channel near the original cross point of the wires, as shown in the images in Figure 5. This leads to electron acceleration across the gap(s) and the generation of energetic electrons. These, in turn, interact with the dense plasmas that can be seen above the original cross point in the four images in Figure 5 and produce X-ray radiation (bremsstrahlung) in the 10 - 100 $\mathrm{keV}$ range. Spectral and temporal specifics of the electron-beam-induced radiation depend on both the X-pinch material and the generator parameters [9] [10] [22]. This component of X-pinch radiation in our experiments has a source size of $0.1-1 \mathrm{~mm}$.

The final stage of hybrid X-pinch (HXP) development is similar in many aspects to that of a standard X-pinch. In the HXP, fast expansion of the hot spot plasma, expansion and clearing of the micropinch plasma channel, and electron acceleration also occur after the X-ray burst. However, at the very end, due to the small gap between the conical electrodes (Figure 3(d)), the gap is bridged by the electrode plasma [11]-[13]. This means that conditions for the acceleration of electrons are present for significantly less time than in a standard X-pinch, and the source size of the $10-100 \mathrm{keV} \mathrm{X-rays} \mathrm{is} \mathrm{smaller} \mathrm{than} \mathrm{in} \mathrm{standard} \mathrm{X-pinches} \mathrm{in} \mathrm{all} \mathrm{directions} \mathrm{[12].} \mathrm{The}$ parameters of the radiation sources in hybrid X-pinches are summarized in Table 1, Figure 6.

\section{Results and Discussion}

X-ray imaging of different biological objects has been developed in parallel with studying the physics of the $\mathrm{X}$-pinch itself and its characteristics as an X-ray source. The objects are imaged in different media and with different magnification based on the objectives, size and density of the object.

For imaging small objects with high magnification and high spatial resolution, the radiation from the $\sim 1 \mu \mathrm{m}$ hot spots obtained with standard Mo-wire X-pinches is used. One of the objects studied with a member of the agricultural college at Cornell University is a beet seed. The seed and its shell are both high density. The image is to be done without damaging the seed [23]. To obtain this image, the more energetic X-rays from the hot spot are used (Figure 7).

In a study of small insects with parasites in them, the insects are in formalin solution. For this purpose of a sealed chamber is built that can be placed in vacuum with the insects in it. Radiographs are obtained using hot spot radiation through thin vacuum windows in the box, as shown in Figure 8.

In the images in both Figure 7 and Figure 8, $\mu \mathrm{m}$-scale elements of the internal structure are captured because of the phenomenon of phase contrast discussed above. For imaging of relatively large biological objects, the radiation from the hot spot is too low energy, so we use the higher energy $\mathrm{X}$-rays produced by the electron beams. In this case, the object may be outside the vacuum chamber in air or liquid. For example, in Figure 9, we 

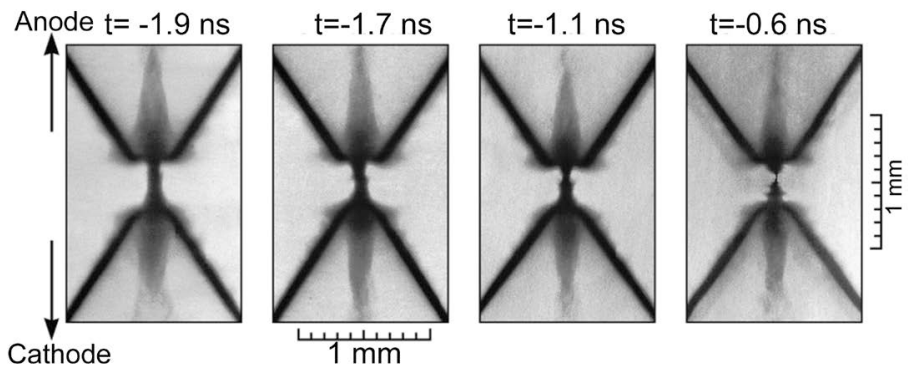

Figure 4. Radiographs of two-wire (17 $\mu \mathrm{m}$ diam. Mo) X-pinches showing the development stages of the micropinch formation at 4 times in the 2 ns immediately before hot spot formation. Each image was obtained in radiation from a similar X-pinch in the configuration shown in Figure 3(c). The indicated times are before the X-pinch in the image emitted its X-ray burst.
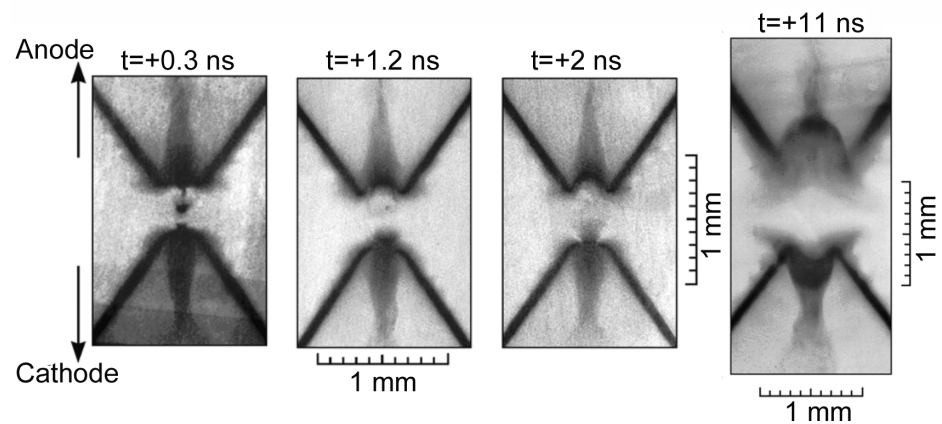

Figure 5. Radiographs of two-wire (17 $\mu \mathrm{m}$ diam. Mo) X-pinches illustrate the breaking of the plasma channel near the original wire cross point and the formation of conditions for electron acceleration and radiation. Each image was obtained in radiation from a similar X-pinch in the configuration shown in Figure 3(c). The indicated times are after the X-pinch in the image emitted its X-ray burst.

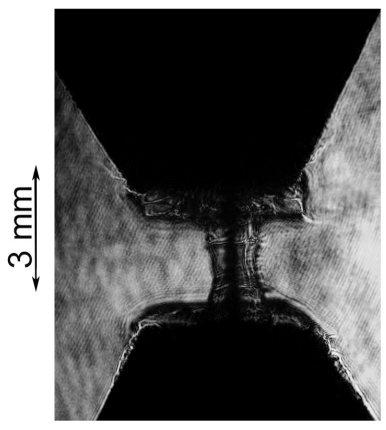

(a)

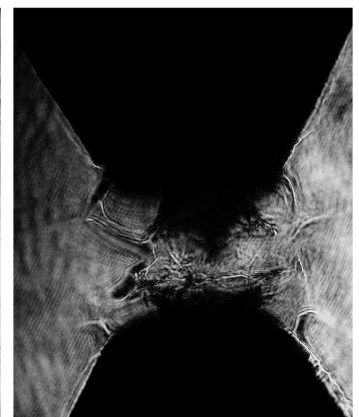

(b)

Figure 6. Laser shadowgraphs of a hybrid X-pinch with $12.7 \mu \mathrm{m}$ diameter Mo wire recorded (a) 3 ns before and (b) 2 ns after the emission burst. The laser pulse duration is about $0.2 \mathrm{~ns}$. (a) $t=-3 \mathrm{~ns}$; (b) $t=+2 \mathrm{~ns}$.

show a radiograph of a frog in water that is outside of the vacuum chamber. Small features of the frog inner structure are clearly seen on the figure.

The use of the electron beam-generated radiation from hybrid X-pinches, which has a smaller source size than from standard X-pinches, allows the use of point-projection imaging with small magnification to obtain an image with higher spatial resolution, as shown in Figure 10.

The large field of view of the point projection method allows images of multiple objects with a single burst of radiation. 


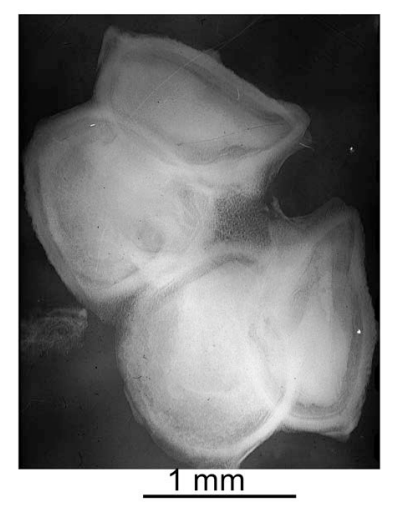

Figure 7. Point-projection image of a beet seed in its shell obtained in the hot spot radiation from a 4-wire Mo $\mathrm{X}$-pinch using X-rays with energy $>4 \mathrm{keV}$. The imaging geometry gave an object to film magnification 1:8.

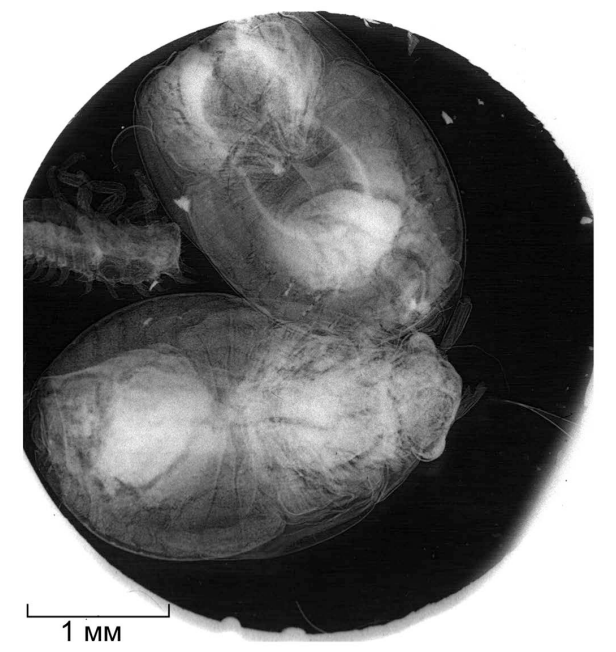

Figure 8. Point-projection image of bugs in formalin obtained in hot spot radiation from a 4-wire Mo X-pinch using X-rays with energy $>3 \mathrm{keV}$. The object-film geometry gave magnification 1:6.
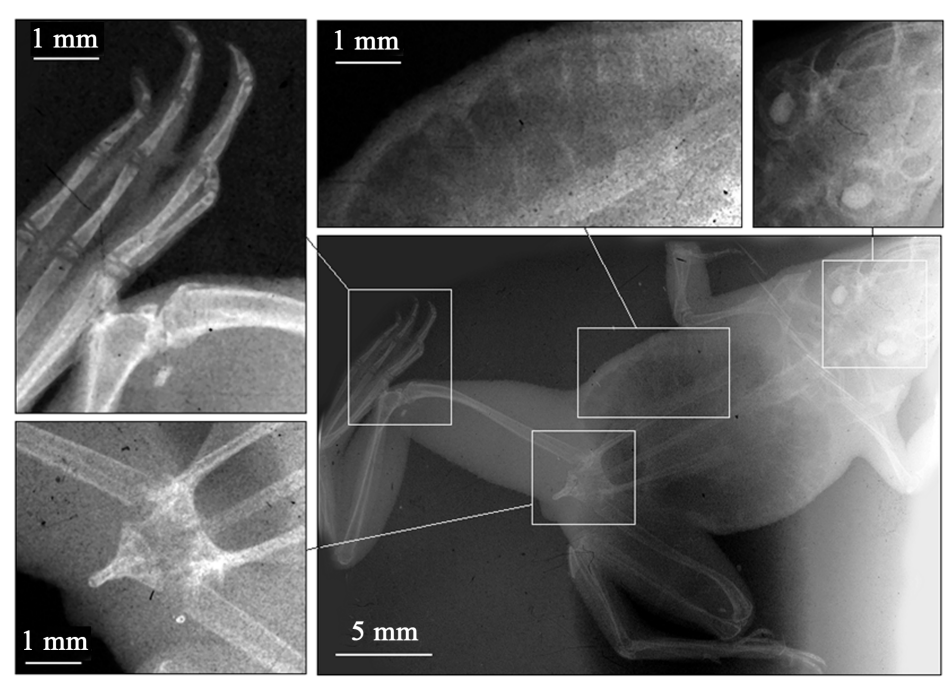

Figure 9. Radiograph of a live dwarf frog obtained with the radiation from a 4-wire, $25 \mu \mathrm{m}$ Mo X-pinch using a 5 $\mu \mathrm{m}$ Ta filter and $24 \mu \mathrm{m} \mathrm{Al}$ filter (X-ray energy $8-10 \mathrm{keV}$ and $>12 \mathrm{keV}$ ). The film was placed close behind the object giving a magnification of 1:1.05 (The image was obtained in experiments of B. M. Song). 


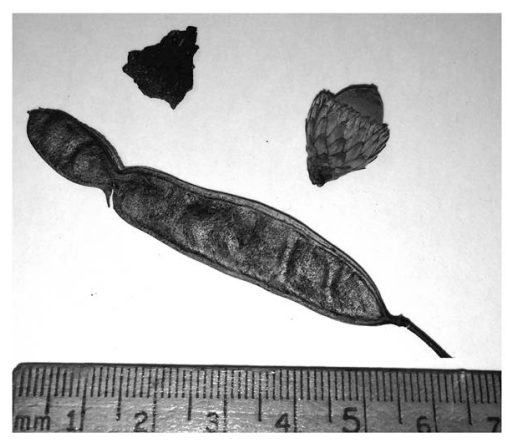

(a)

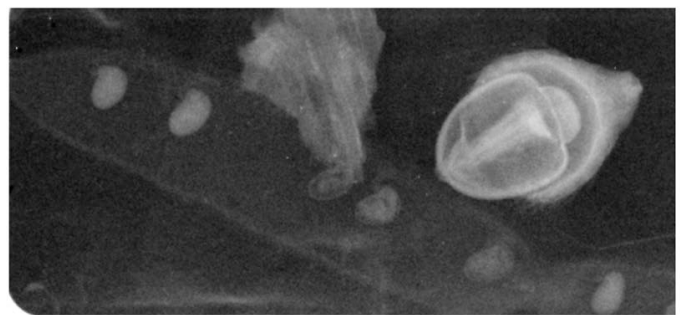

$10 \mathrm{~mm}$

(b)

Figure 10. (a) Photograph of a piece of bark, an acorn and a seed pod from a tree; (b) An image in the electron-beamgenerated radiation with energy $>8 \mathrm{keV}$ from a hybrid X-pinch of the objects in (a) is shown. The image was recorded on an Image Plate with a geometric magnification of 1:2.5.

Table 1. Hybrid X-pinch (HXP) and standard X-pinch (SXP) source parameters (E = X-ray energy).

\begin{tabular}{|c|c|c|c|}
\hline Source parameters & $\begin{array}{l}\text { Hot spot radiation } \\
\text { in SXP and HXP }\end{array}$ & $\begin{array}{l}\text { Electron beam } \\
\text { radiation in SXP }\end{array}$ & $\begin{array}{l}\text { Electron beam } \\
\text { radiation in HXP }\end{array}$ \\
\hline Source size & $0.5-10 \mu \mathrm{m}$ & $100-1000 \mathrm{~mm}$ & $40-150 \mu \mathrm{m}$ \\
\hline $\mathrm{X}$-ray burst duration & $0.01-1 \mathrm{~ns}$ & $5-30 \mathrm{~ns}$ & $2-5$ ns \\
\hline Energy of X-rays & $1<\mathrm{E}<10 \mathrm{keV}$ & $8<\mathrm{E}<200 \mathrm{keV}$ & $8<\mathrm{E}<200 \mathrm{keV}$ \\
\hline Radiated energy in the burst & $0.01-2 \mathrm{~J}$ & $1-10 \mathrm{~J}$ & no measurement \\
\hline
\end{tabular}

To summarize very briefly, X-pinches can be a simple and cheap X-ray source over a wide range of X-ray energies for point-projection radiography of a variety of biological or medical objects. Comparisons of the $\mathrm{X}$-pinch based source of radiation with other sources are possible to find elsewere [8] [24].

\section{Acknowledgements}

This research was supported by the National Nuclear Security Administration Stewardship Sciences Academic Programs under Department of Energy Cooperative Agreement No. DE-NA0001836.

\section{References}

[1] Zakharov, S.M., Ivanenkov, G.V., Kolomenskii, A.A., Pikuz, S.A., Samokhin, A.I. and Ulshmid, J. (1982) Wire XPinch in a High Current Diode. Soviet Technical Physics Letters, 8, 456-457.

[2] Kalantar, D.H. and Hammer, D.A. (1995) The X-Pinch as a Point Source of X Rays for Backlighting. Review of Scientific Instruments, 66, 779-781. http://dx.doi.org/10.1063/1.1146219

[3] Shelkovenko, T.A., Sinars, D.B., Pikuz, S.A. and Hammer, D.A. (2001) Radiographic and Spectroscopic Studies of X Pinch Plasma Implosion Dynamics and X-Ray Burst Emission Characteristics. Physics of Plasmas, 6, 1305-1318. http://dx.doi.org/10.1063/1.1351553

[4] Pikuz, S.A., Shelkovenko, T.A. and Hammer, D.A. (2015) X-Pinch. Plasma Physics Reports, 41, $291-342$. http://dx.doi.org/10.1134/S1063780X15060045 
[5] Pikuz, S.A., Shelkovenko, T.A., Mingaleev, A.R., Romanova, V.M., Song, B.M., Chandler, K.M., Mitchell, M.D. and Hammer, D.A. (2005) The X-Pinch as an X-Ray Source for Point-Projection Radiography. The International Society for Optical Engineering, International Conference on Charged and Neutral Particles Channeling Phenomena, 5974, $59740 \mathrm{~T} 1$.

[6] Shelkovenko, T.A., Pikuz, S.A., Douglass, J.D., Greenly, J.B. and Hammer, D.A. (2006) Multiwire X Pinches at 1-MA Current on the COBRA Pulsed Power Generator. IEEE Transactions on Plasma Science, 34, 2336-2241. http://dx.doi.org/10.1109/TPS.2006.878362

[7] Shelkovenko, T.A., Pikuz, S.A., Cahill, A.D., Knapp, P.F., Hammer, D.A., Sinars, D.B., Tilikin, I.N. and Mishin, S.N. (2010) Hybrid X-Pinch with Conical Electrodes. Physics of Plasmas, 17, 112707-1-112707-5. http://dx.doi.org/10.1063/1.3504226

[8] Shelkovenko, T.A., Pikuz, S.A., Song, B.M., Chandler, K.M., Mitchell, M.D., Hammer, D.A., Ivanenkov, G.V., Mingaleev, A.R. and Romanov, V.M. (2005) Electron-Beam-Generated X-Rays from X Pinches. Physics of Plasmas, 12, 033102-033108. http://dx.doi.org/10.1063/1.1849798

[9] Shelkovenko, T.A., Pikuz, S.A., Mingaleev, A.R., Agafonov, A.V., Romanova, V.M., Ter-Oganes’yan, A.E., Tkachenko, S.I., Blesener, I.C., Mitchell, M.D., Chandler, K.M., Kusse, B.R. and Hammer, D.A. (2008) Accelerated Electrons and Hard X-Ray Emission from X-Pinches. Plasma Physics Reports, 34, 754-770. http://dx.doi.org/10.1134/S1063780X08090109

[10] Shelkovenko, T.A., Pikuz. S.A., Hoyt, C.L., Cahill, A.D., Hammer, D.A., Tilikin, I.N., Mingaleev, A.R. and Agafonov, A.V. (2014) Hard X-Rays from Hybrid X Pinches. AIP Conference Proceedings, 1639, 108-111. http://dx.doi.org/10.1063/1.4904788

[11] Shelkovenko, T.A., Pikuz, S.A., Mishin, S.N., Mingaleev, A.R., Tilikin, I.N., Knapp, P.F., Cahill, A.D., Hoyt, C.L. and Hammer, D.A. (2010) Hybrid X-Pinches. Plasma Physics Reports, 38, 359-381. http://dx.doi.org/10.1134/S1063780X1204006X

[12] Shelkovenko, T.A., Tilikin, I.N., Ivanenkov, G.V., Stepniewski, W., Mingaleev, A.R., Romanova, V.M., Agafonov, A.V., Cahill, A.D., Hoyt, C.L., Gourdain, P.A., Hammer, D.A. and Pikuz, S.A. (2015) Dynamics of Hybrid X-Pinches. Plasma Physics Reports, 41, 52-70. http://dx.doi.org/10.1134/S1063780X15010031

[13] Song, B.M., Pikuz, S.A., Shelkovenko, T.A. and Hammer, D.A. (2002) Small Size X-Pinch Radiation Source for Application to Phase-Contrast X-Ray Radiography of Biological Specimens. IEEE Nuclear Science Symposium and Medical Imaging Conference, 2, 868-872.

[14] Song, B.M., Pikuz, S.A., Shelkovenko, T.A. and Hammer, D.A. (2005) Determination of the Size and Structure of an X Pinch X-Ray Source from the Diffraction Pattern Produced by Microfabricaterd Slits. Applied Optics, 44, $2349-2358$. http://dx.doi.org/10.1364/AO.44.002349

[15] Greenly, J.B., Douglass, J.D., Hammer, D.A., Kusse, B.R., Glidden, S.C. and Sanders, H.D. (2008) A 1 MA, Variable Risetime Pulse Generator for High Energy Density Plasma Research. Review of Scientific Instruments, 79, 073501. http://dx.doi.org/10.1063/1.2949819

[16] Beg, F.N., Krushelnik, K., Lichtsteiner, P., Meakins, A., Kennedy, A., Kajumba, N., Burt, G. and Dangor, A.E. (2003) Table-Top X-Pinch for X-Ray Radiography. Applied Physics Letters, 82, 4602-4604. http://dx.doi.org/10.1063/1.1584782

[17] Mesyats, G.A., Shelkovenko, T.A., Ivanenkov, G.V., Agafonov, A.V., Savinov, S.Y., Pikuz, S.A., Tilikin, I.N., Tkachenko, S.I., Chaikovskii, S.A., Ratakhin, N.A., Fedushchak, V.F., Oreshkin, V.I., Fedunin, A.V., Russkikh, A.G., Labetskaya, N.A., Artemov, A.P., Hammer, D.A. and Sinars, D.B. (2010) X-Pinch Source of Subnanosecond Soft X-Ray Pulses Based on Small-Sized Low-Inductance Current Generator. Journal of Experimental and Theoretical Physics, 111, 363-370. http://dx.doi.org/10.1134/S1063776110090049

[18] Artyomov, A.P., Fedyunin, A.V., Chaikovsky, S.A., Zhigalin, A.S., Oreshkin, V.I., Ratakhin, N.A. and Rousskikh, A.G. (2013) A Double-Frame Nanosecond Soft X-Ray Backlighting System Based on X-Pinches. Instruments and Experimental Techniques, 56, 66-71. http://dx.doi.org/10.1134/S0020441213010028

[19] Pikuz, S.A., Shelkovenko, T.A., Sinars, D.B. and Hammer, D.A. (2006) Temporal Characteristics of X-Ray Emission from X-Pinches. Plasma Physics Report, 32, 1020-1033. http://dx.doi.org/10.1134/S1063780X06120051

[20] Pikuz, S.A., Shelkovenko, T.A., Mingaleev, A.R., Hammer, D.A. and Neves, H.P. (1999) Density Measurements in Txploding Wire-Initiated Plasmas Using Tungsten Wires. Physics of Plasmas, 6, 4272-4283. http://dx.doi.org/10.1063/1.873695

[21] Song, B.M., Shelkovenko, T.A., Pikuz, S.A., Mitchell, M.A., Chandler, K.M. and Hammer, D.A. (2004) X Pinch X-Ray Radiation Above $8 \mathrm{keV}$ for Application to High-Resolution Radiography of Biological Specimens. IEEE Transactions on Nuclear Science, 51, 2514-2519. http://dx.doi.org/10.1109/TNS.2004.835738

[22] Shelkovenko, T.A., Pikuz, S.A., Blesener, I.C., McBride, R.D., Bell, K.S., Hammer, D.A., Agafonov, A.V., Romanova, 
V.M. and Mingaleev, A.R. (2008) Measurements of High-Current Electron Beams from X Pinches and Wire Array Z Pinches. Review of Scientific Instruments, 79, 10E316. http://dx.doi.org/10.1063/1.2957920

[23] Tailor, A.G., Goffinet, M.C., Pikuz, S.A., Shelkovenko, T.A., Mitchell, M.D., Chandler, K.M. and Hammer, D.A. (2003) Physico-Chemical Factors Influence Beet (Beta vulgris L.) Seed Germination. In: Nicolás, G., Bradford, K.J., Côme, D. and Pritchard, H.W., Eds., The Biology of Seeds: Recent Research Advances, CAB International, Wallingford, 433-440.

[24] Shekovenko, T.A., Pikuz, S.A. and Hammer, D.A. (2015) Point-Projection Radiography of Plasma and Biological Objects in an X-Pinch Radiation. Plasma Physics Reports. (in press) 